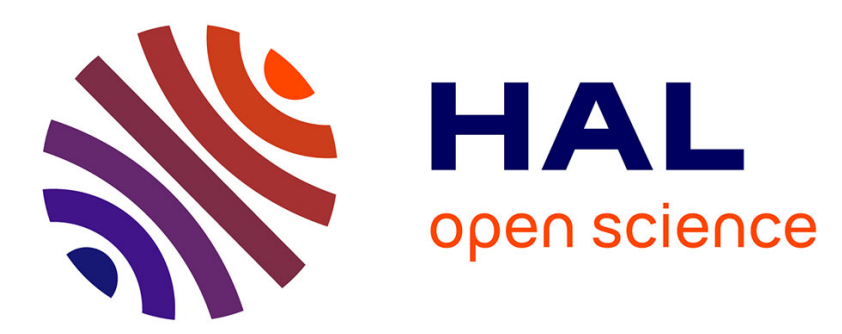

\title{
Analyse théorique et expérimentale de l'effort et du couple dans le laminage des matériaux superplastiques
}

\author{
A.R. Ragab, B. Baudelet
}

\section{To cite this version:}

A.R. Ragab, B. Baudelet. Analyse théorique et expérimentale de l'effort et du couple dans le laminage des matériaux superplastiques. Revue de Physique Appliquée, 1978, 13 (10), pp.495-501. 10.1051/rphysap:019780013010049500 . jpa-00244480

HAL Id: jpa-00244480

https://hal.science/jpa-00244480

Submitted on 1 Jan 1978

HAL is a multi-disciplinary open access archive for the deposit and dissemination of scientific research documents, whether they are published or not. The documents may come from teaching and research institutions in France or abroad, or from public or private research centers.
L'archive ouverte pluridisciplinaire HAL, est destinée au dépôt et à la diffusion de documents scientifiques de niveau recherche, publiés ou non, émanant des établissements d'enseignement et de recherche français ou étrangers, des laboratoires publics ou privés. 


\author{
Classification \\ Physics Abstracts \\ $46.20-46.30-46.30 \mathrm{~J}$
}

\title{
ANALYSE THÉORIQUE ET EXPÉRIMENTALE DE L'EFFORT ET DU COUPLE DANS LE LAMINAGE DES MATÉRIAUX SUPERPLASTIQUES
}

\author{
A. R. RAGAB \\ Mechanical Design and Production Department, Cairo University, Egypt \\ et \\ B. BAUDELET \\ Laboratoire de Physique et de Technologie des Matériaux \\ Associé au C.N.R.S. No 155, Université de Metz, France
}

(Reçu le 7 avril 1978, révisé le 26 juin 1978, accepté le 28 juin 1978)

\begin{abstract}
Résumé. - L'effort et le moment du couple appliqués aux cylindres lors du laminage d'un matériau sensible à la vitesse de déformation sont calculés en tenant compte de l'influence des variations de la vitesse de déformation entre les cylindres sur la contrainte d'écoulement. Les efforts sur les cylindres ont été mesurés au cours du laminage à la température ampiante de l'alliage superplastique eutectique $\mathrm{Pb}-\mathrm{Sn}$ avec des cylindres rectifiés et bien lubrifiés et pour différentes vitesses. Les charges mesurées sont en bon accord avec les valeurs théoriques estimées. Une analyse théorique démontre qu'une réduction de la force appliquée aux cylindres peut être obtenue en laminant des alliages dans des conditions où un comportement superplastique peut se manifester. Ceci semble être d'un intérêt industriel pour laminer des alliages particulièrement résistants au laminage et ceux qui présentent un comportement superplastique temporaire sous de grandes vitesses de sollicitation.
\end{abstract}

\begin{abstract}
The roll load and torque necessary to roll a strain-rate sensitive material have been theoretically analysed allowing for the strain-rate distribution and its influence on the flow stress in the rollgap. The roll loads have been measured during room temperature rolling of superplastic lead-tin eutectic alloy between smooth lubricated rolls at various speeds. The measured loads agree satisfactorily with the theoretically estimated ones. Theoretical analysis demonstrates that savings in roll loads could be achieved by rolling alloys in their superplastic state at the appropriate rolling speeds. This seems to be of industrial interest for rolling hard-to-roll alloys and in the exploitation of the phenomenon of temporary superplasticity.
\end{abstract}

Introduction. - Les théories du laminage des bandes ont pour but d'estimer correctement l'effort et le couple auxquels sont soumis les cylindres à partir de la géométrie des cylindres, de la résistance mécanique de ceux-ci et du matériau laminé.

Lors du laminage à chaud pour lequel la résistance à la déformation dépend fortement de la vitesse, les approches théoriques connues sont fondées sur l'hypothèse d'une contrainte d'écoulement du matériau constante durant la passe de laminage. L'utilisation d'une valeur moyenne de la contrainte d'écoulement (obtenue à partir des courbes contraintevitesse dans le domaine approprié des vitesses) fut alors proposée comme la seule méthode pratique permettant de tenir compte de l'effet de la vitesse sur la contrainte d'écoulement [1] et [2].

Cette hypothèse doit introduire de faibles erreurs dans le calcul de la force et du moment du couple lors du laminage à chaud d'alliages conventionnels, pour lesquels le coefficient de sensibilité $m$ à la vitesse de déformation est faible. En revanche, cette erreur doit être beaucoup plus grande lors du laminage d'alliages dans des conditions de superplasticité. En effet, ces alliages sont caractérisés par une valeur élevée du coefficient $m$, valeur qui peut approcher celle des liquides visqueux tels que le verre fondu et les polymères thermoplastiques à température élevée.

Les résultats théoriques, estimés par cette méthode, 
sont comparés aux résultats expérimentaux obtenus lors du laminage lubrifié de l'alliage superplastique plomb-étain. Le laminage a été réalisé à différentes vitesses sur une machine de laboratoire.

\section{Nomenclature}

$t \quad$ Temps

$h \quad$ Epaisseur de la tôle pour un angle $\varphi$

$h_{1}, h_{2}, h_{\mathrm{N}}$ Epaisseur de la tôle à l'entrée du laminoir, à la sortie et à l'angle neutre respectivement

$\varphi \quad$ Angle d'un arc de contact

$\varphi_{\mathrm{N}}, \varphi_{1} \quad$ Angle neutre et angle total de contact

$R \quad$ Rayon du cylindre

$V_{\mathrm{N}} \quad$ Vitesse périphérique de chaque cylindre

$\delta \quad$ Réduction d'épaisseur $=h_{1}-h_{2}$

$r \quad$ Taux de réduction $=.\left(h_{1}-h_{2}\right) / h_{1}$

$L \quad$ Longueur de l'arc de contact

$m \quad$ Coefficient de sensibilité de la contrainte

à la vitesse de déformation

$\sigma_{0}, \dot{\varepsilon}_{0} \quad$ Paramètres liés aux matériaux

$\sigma_{\mathrm{e}} \quad$ Contrainte équivalente

$\dot{\varepsilon}_{\mathrm{e}} \quad$ Vitesse de déformation équivalente

$\mu \quad$ Coefficient de frottement

$p \quad$ Contrainte horizontale dans l'espace compris entre les cylindres

$S \quad$ Pression normale au cylindre

$P, T \quad$ Respectivement effort et moment du couple appliqués aux cylindres par unité de largeur (effort et moment du couple spécifiques);

1. Analyse théorique. - Les hypothèses de base habituelles dans les théories du laminage des tôles sont conservées dans la présente analyse : conditions de déformation plane; arc de contact circulaire; déformation homogène et déformation élastique négligeable.

Les conditions de frottement entre le cylindre et le matériau laminé sont supposées du type coulombien, avec un coefficient de frottement constant. Ce type de frottement a été utilisé dans l'analyse de problèmes de laminage à froid. De plus, des études récentes [3] et [4] ont montré que des conditions de frottement de type coulombien prévalent dans la plupart des conditions de laminage à chaud.

Lors du laminage de tôles et de bandes, les conditions de déformation planes sont réunies.

En considérant la géométrie (Fig. 1) de l'espace compris entre les cylindres, on voit facilement que la vitesse de déformation locale équivalente est donnée par [1]

$$
\dot{\varepsilon}_{\mathrm{e}}=\frac{4 V_{\mathrm{N}} h_{\mathrm{N}}}{\sqrt{3 R}} \frac{\sqrt{h-h_{2}}}{h^{2}}
$$

La figure 2 donne la variation du paramètre $\dot{\varepsilon}_{\mathrm{e}} /\left(4 V_{\mathrm{N}} h_{\mathrm{N}} / \sqrt{3 R}\right)$ en fonction du rapport $\left(h-h_{2}\right) / \delta$ pour une géométrie de laminage donnée et pour différents taux de réduction. La vitesse de déformation

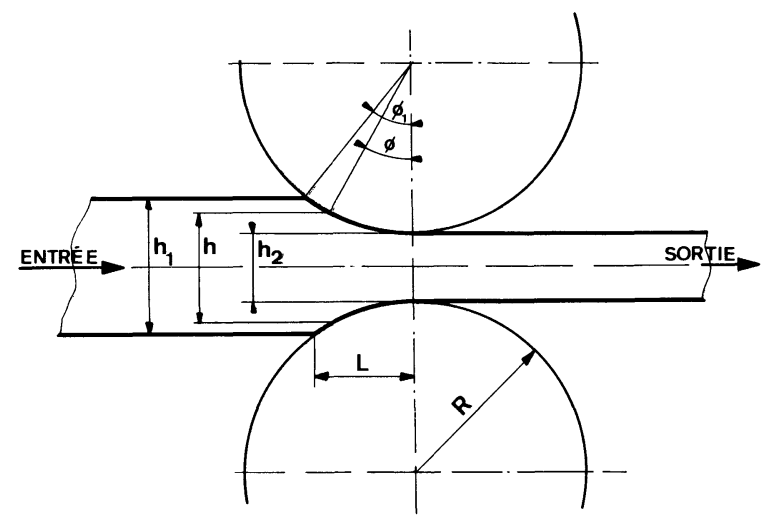

FIG. 1. - Géométrie du laminage.

[Rolling geometry.]

est maximale en un point donné entre les cylindres, et sa position dépend du taux de réduction de la passe. Près du plan de sortie, la vitesse décroît rapidement jusqu'à zéro. Cependant, sur plus de $99,5 \%$ de la zone en cours de réduction d'épaisseur, la vitesse ne varie pas de plus d'un ordre de grandeur pour les taux de réduction indiqués figure 2 .

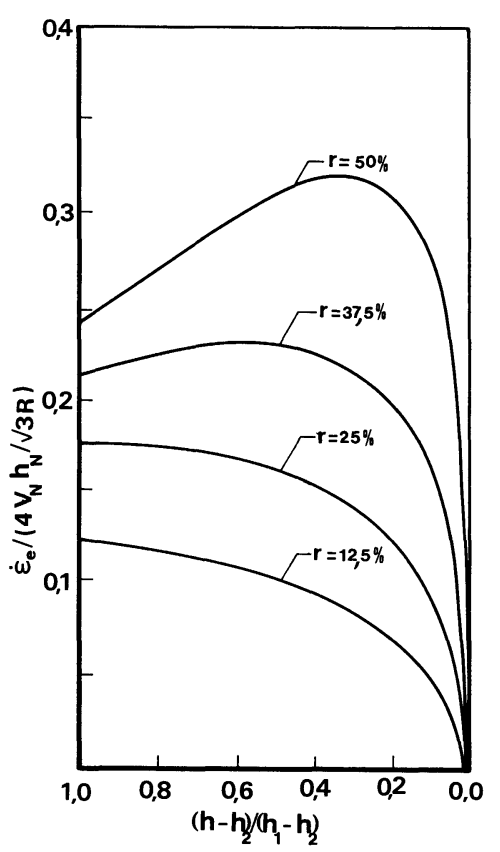

Fig. 2. - Distribution des vitesses de déformation le long de l'arc de contact pour différents taux de réduction; $R=90 \mathrm{~mm}$, $h_{1}=2 \mathrm{~mm}$.

[Distribution of strain-rate through the arc of contact for various reductions, $R=90 \mathrm{~mm}, h_{1}=2 \mathrm{~mm}$.]

Pour les alliages qui présentent un comportement superplastique, dans des conditions isothermes et dans un domaine limité de vitesse de déformation, une loi puissance du type

$$
\sigma_{\mathrm{e}}=\sigma_{0}\left(\dot{\varepsilon}_{\mathrm{e}} / \dot{\varepsilon}_{0}\right)^{m}
$$

représente approximativement le comportement mécanique de ces alliages [5]. Le paramètre $\dot{\varepsilon}_{0}$ est habituel- 
lement pris égal à l'unité; $\sigma_{0}$ est alors la contrainte d'écoulement du matériau à la vitesse de déformation unitaire. L'équation différentielle de base qui permet de déterminer la contrainte horizontale $p$ entre les cylindres de laminoir lorsqu'on tient compte du coefficient de sensibilité $m$ est donnée par l'expression (cf. annexe).

$$
\begin{aligned}
& \frac{\mathrm{d} p}{\mathrm{~d} \varphi} \mp \frac{2 \mu R}{h_{2}+R \varphi^{2}} p= \\
& =2 K \sigma_{0} R^{1+m / 2}\left(h_{2}+R \varphi^{2}\right)^{-2 m-1} \cdot(\varphi \pm \mu) \varphi^{m} .
\end{aligned}
$$

Les signes supérieurs dans cette équation correspondent à la sortie du laminoir et les signes inférieurs à l'entrée. Les équations différentielles (3) ne sont pas susceptibles d'intégration analytique. Elles sont donc intégrées numériquement en prenant pour conditions $p=0$ lorsque $\varphi=0$ et $\varphi=\varphi_{1}$ [6]. Une fois la distribution des contraintes horizontales $p(\varphi)$ obtenue, la distribution des pressions normales au cylindre $S(\varphi)$ a pu être déterminée grâce à la relation (cf. annexe)

$$
\begin{aligned}
& S=p+K \sigma_{0}\left(h-h_{2}\right)^{m / 2} h^{-2 m} \\
& K=\frac{2}{\sqrt{3}}\left(\frac{4 V_{\mathrm{N}} h_{\mathrm{N}}}{\dot{\varepsilon}_{0} \sqrt{3 R}}\right)^{m} .
\end{aligned}
$$

L'effort appliqué au cylindre par unité de largeur, appelé dans la suite effort spécifique sur le cylindre; est donné par l'expression :

$$
\begin{aligned}
P / \frac{2 \sigma_{0}}{\sqrt{3}} & =\left(\frac{4 V_{\mathrm{N}} h_{\mathrm{N}}}{\sqrt{3 R} \dot{\varepsilon}_{0}}\right)^{m} \times \\
& \times R\left[\int_{\varphi_{\mathrm{N}}}^{\varphi_{1}}\left(\frac{S}{K \sigma_{0}}\right) \mathrm{d} \varphi+\int_{0}^{\varphi_{\mathrm{N}}}\left(\frac{S}{K \sigma_{0}}\right) \mathrm{d} \varphi\right] .
\end{aligned}
$$

L'expression à gauche du signe égal $P / \frac{2 \sigma_{0}}{\sqrt{3}}$ est analogue au rapport bien connu $P / 2 k$ qui intervient dans l'analyse du laminage des matériaux parfaitement plastiques ( $k$ représente dans ce cas, la contrainte d'écoulement en cisaillement). L'équation (6) indique que l'effort exercé sur le cylindre est fonction de la vitesse de rotation de ce dernier et de la sensibilité à la vitesse du matériau.

L'effort à appliquer aux cylindres de laminoir pour des matériaux ayant une même valeur $\sigma_{0}$, dépend du terme $K$ défini par l'expression (5), c'est-àdire de la vitesse de laminage et du coefficient de sensibilité à la vitesse de déformation. La figure 3 montre que pour une valeur de $\sigma_{0}$ et un taux de réduction donnés, il existe une certaine vitesse de laminage en dessous de laquelle toute augmentation du coefficient $m$ produit une diminution importante de l'effort à appliquer. Le domaine de vitesse inclut celui où

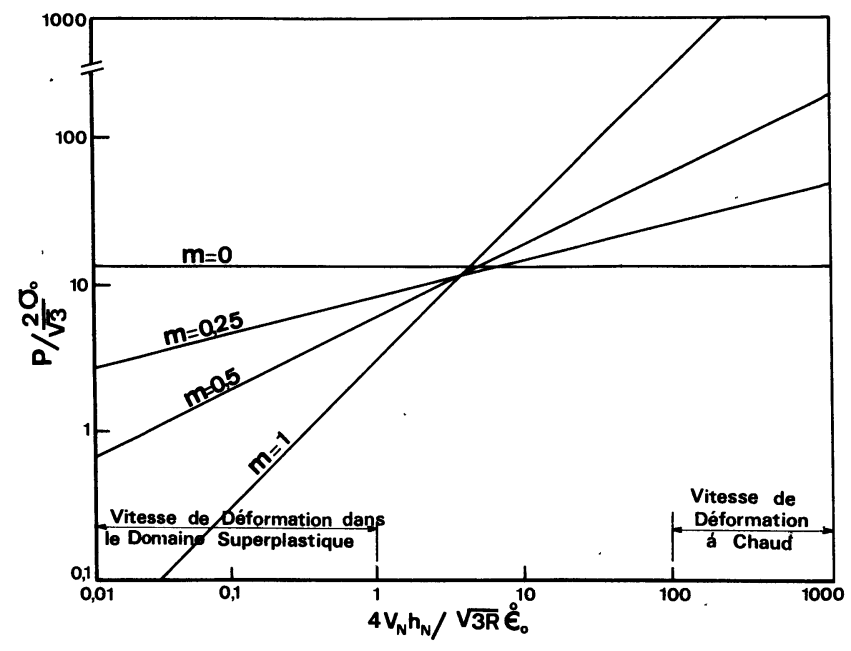

Fig. 3. - Effort spécifique exercé sur les cylindres en fonction de la vitesse du laminoir; $R=90 \mathrm{~mm}, h_{1}=2 \mathrm{~mm}, \mu=0,14$, $r=50 \%$.

[Specific roll load versus roll speed parameter; $R=90 \mathrm{~mm}$, $h_{1}=2 \mathrm{~mm}, \mu=0.14, r=50 \%$.]

le matériau présente un comportement superplastique. En revanche, aux vitesses élevées de laminage, la force à appliquer au cours du laminage croît avec la valeur du coefficient $m$. Ce dernier résultat est en fait une conséquence d'une description mathématique; en réalité, aux vitesses élevées, des valeurs grandes du coefficient $m$ ne peuvent pas être observées en déformation à chaud. Cependant, lorsqu'à une vitesse de déformation supérieure à l'unité, des matériaux ont même contrainte d'écoulement mais des valeurs différentes de coefficient $m$ de sensibilité à la vitesse de déformation, la valeur de $\sigma_{0}$, prise pour $\dot{\varepsilon}$ égal à l'unité, est plus faible pour les matériaux qui présentent une valeur plus élevée du coefficient $m$. Alors, aux valeurs élevées du coefficient $m$ correspondent des forces plus faibles si le laminage est effectué à des vitesses inférieures à celles qui correspondent au point commun. Ce dernier cas pourrait s'appliquer à la superplasticité temporaire [7]

2. Etude expérimentale. - 2.1 MATÉRIAU. - Des plaques d'alliage eutectique plomb-étain ont été coulées puis homogénéisées par un maintien d'environ 20 heures à $130^{\circ} \mathrm{C}$. Ces plaques ainsi homogénéisées ont été ensuite laminées à température ambiante en passes longitudinales et transversales successives jusqu'à un taux de réduction totale de l'épaisseur de $80 \%$. Les bandes ainsi obtenues ont été découpées sous forme de rectangle puis de nouveau homogénéisées à $130{ }^{\circ} \mathrm{C}$ pendant 30 minutes. Ce traitement thermomécanique a permis d'obtenir les grains fins requis pour que le matériau présente un comportement superplastique [8].

La variation de la contrainte d'écoulement avec la vitesse de déformation déterminée par des essais de compression à température ambiante est donnée sur la figure. En négligeant l'influence de la déformation $\varepsilon$, sur la contrainte d'écoulement durant 


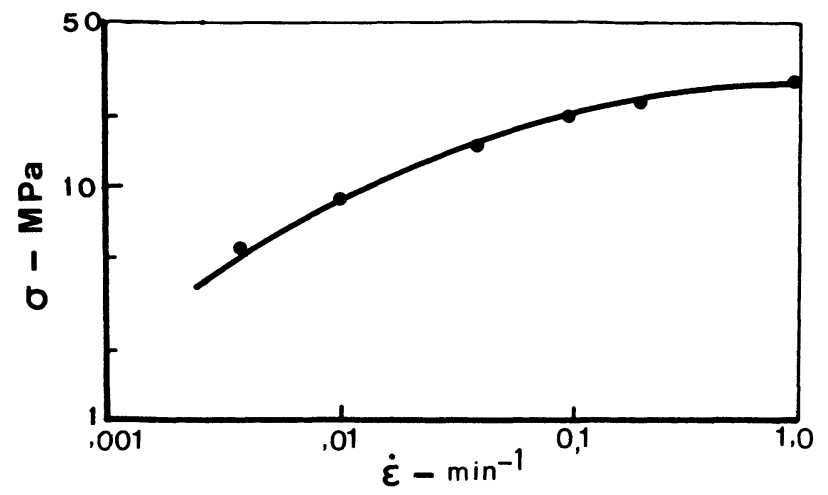

Fig. 4. - Relation contrainte d'écoulement-vitesse de déformation pour l'alliage superplastique eutectique $\mathrm{PbSn}$.

[Flow stress versus strain-rate for superplastic eutectic $\mathrm{Pb}-\mathrm{Sn}$ alloy.]

l'essai de compression, une relation du type $\sigma_{\mathrm{e}}=f\left(\dot{\varepsilon}_{\mathrm{e}}\right)$ peut être utilisée pour représenter le comportement du matériau. Sur un domaine de vitesse de déformation limité (par exemple un ordre de grandeur), la fonction puissance (2) représente la variation observée sur la figure 4 . La valeur du coefficient de sensibilité à la vitesse $m$ varie ainsi de 0,15 à 0,6 selon l'ordre de grandeur de la vitesse sur lequel la fonction puissance a été utilisée.

2.2 ESSAIS DE LAMINAGE. - Le laminage des bandes d'alliage plomb-étain superplastique a été effectué sur un laminoir de type duo équipé de deux cylindres rectifiés de $125 \mathrm{~mm}$ de diamètre. L'effort appliqué aux cylindres a été mesuré par une cellule de charge placée entre les vis supportant le cylindre du haut et leurs butées supérieures. Cette cellule de charge était constituée de deux cylindres en alliage d'aluminium sur lequel étaient fixées deux jauges de contrainte incorporées dans un pont de Wheatstone. Le laminoir était doté d'un variateur de vitesse. Les vitesses de rotation du cylindre ont été maintenues suffisamment faibles pour que les vitesses de déformation appliquées se trouvent tout à fait dans le domaine où le comportement est superplastique. Tous les essais de laminage ont été effectués en régime lubrifié par une huile minérale. La détermination expérimentale de l'angle maximum [9] pour engager librement la tôle entre les cylindres du laminoir a été employée pour déterminer le coefficient de frottement. Ce coefficient a été trouvé égal à 0,13 , valeur relativement élevée mais qui peut être due aux faibles vitesses de laminage [4].

2.3 Résultats. - Les bandes d'alliage plombétain superplastique d'épaisseur variant entre 4,5 à $7,7 \mathrm{~mm}$ ont été laminées à des vitesses variables dans un domaine s'étendant sur deux ordres de grandeur. L'épaisseur relativement forte des bandes a été choisie afin que les vitesses de déformation moyennes dans la zone en cours de déformation soient suffisamment faibles pour déformer le matériau dans le domaine superplastique.

La figure 5 donne des résultats typiques des mesures d'effort sur les cylindres, pour différentes vitesses de rotation des cylindres et pour les taux de réduction de $12,1 \%$ et $21,1 \%$.

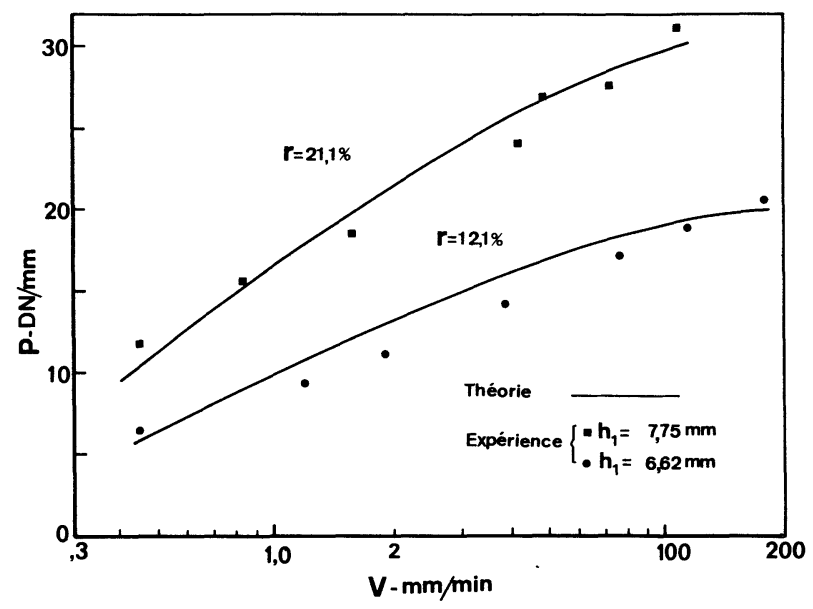

Fig. 5. - Comparaison entre les valeurs calculées et mesurées des efforts spécifiques exercés sur les cylindres du laminoir pour différentes vitesses des cylindres; $R=62,5 \mathrm{~mm}, \mu=0,13$.

[Comparison between calculated and measured specific roll loads at different rolling speeds ; $R=62.5 \mathrm{~mm}, \mu=0.13$.]

Les lignes continues de la figure 5 représentent les prédictions théoriques lorsqu'on fait intervenir la sensibilité à la vitesse du matériau laminé. Les paramètres $m, \sigma_{0}$ ont été obtenus par l'ajustement d'une simple fonction puissance (2) au domaine de vitesses correspondant à $99,5 \%$ de la réduction d'épaisseur imposée par l'essai de laminage considéré. La variation de l'effort spécifique appliqué au cylindre en fonction de la sensibilité à la vitesse est représentée sur la figure 6. Comme les essais de laminage ont été effectués à des vitesses correspondant au

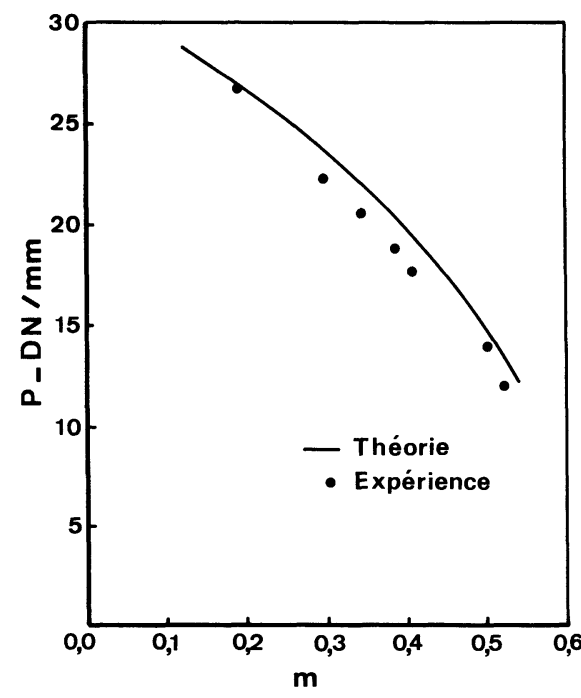

Fig. 6. - Comparaison entre les valeurs calculées et mesurées des efforts spécifiques pour différentes valeurs du coefficient $m$; $R=62,5 \mathrm{~mm}, h_{1}=4,57 \mathrm{~mm}, r=26,7 \%, \mu=0,13$.

[Comparison between calculated and measured specific roll loads for different rate-sensitivities $m ; R=62.5 \mathrm{~mm}, h_{1}=4.57 \mathrm{~mm}$, $r=26.7 \%, \mu=0.13$.] 
domaine superplastique, il apparait que l'effort sur les cylindres décroît lorsque les valeurs du coefficient $m$ croissent. Une comparaison détaillée entre les valeurs calculées et mesurées apparaît sur la figure 7. L'accord entre la théorie et l'expérience est défini par une ligne passant par l'origine et de

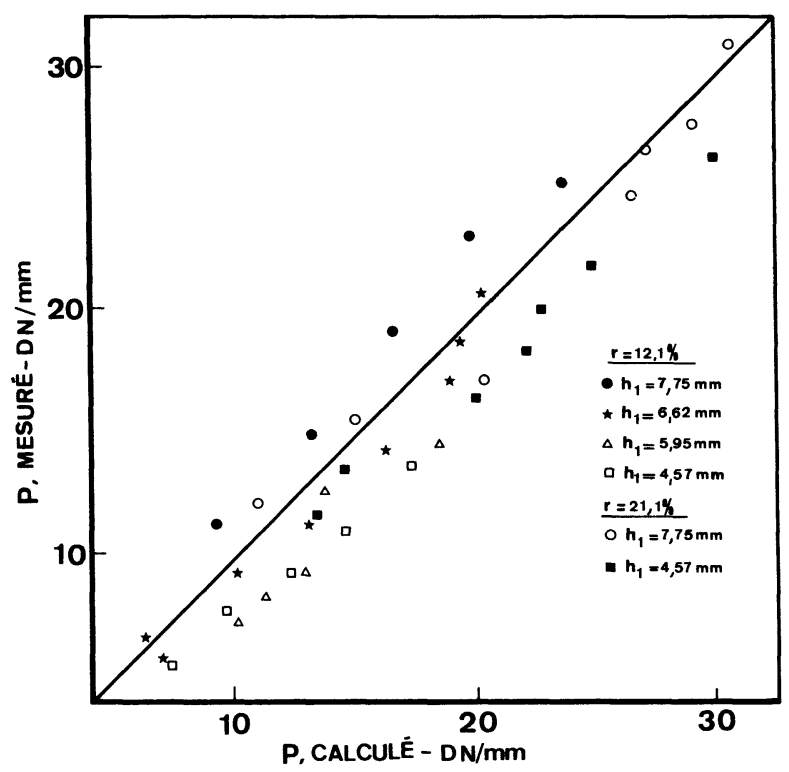

FIG. 7. - Comparaison entre les valeurs calculées et mesurées de l'effort spécifique appliqué au cylindre du laminoir.

[Comparison between calculated and measured specific roll loads $R=62.5 \mathrm{~mm}, \mu=0.13$.] pente $45^{\circ}$. Bien que les points expérimentaux soient très dispersés, comme il est assez fréquent dans de telles comparaisons l'accord entre les prédictions théoriques et les résultats expérimentaux est satisfaisant.

3. Discussion. - Le laminage des matériaux dans leur état superplastique conduit à une réduction considérable de l'effort appliqué et de la puissance requise. Malheureusement, ceci impose de laminer à des vitesses qui peuvent être beaucoup plus faibles (jusqu'à trois ordres de grandeur) que celles utilisées pour le laminage à chaud industriel. Ces vitesses extrêmement lentes représentent la difficulté majeure pour exploiter industriellement la superplasticité d'alliages peu ou moyennement résistants lors du laminage à chaud aux cadences industrielles.

En revanche, pour des alliages à haute résistance tels que les alliages à base de titane et de zirconium, la situation se présente différemment. Ces alliages, dans des conditions conventionnelles, sont difficiles à laminer et des techniques spéciales telles que le laminage sandwich ont dû être utilisées pour réduire les efforts appliqués aux cylindres [9]. Afin d'illustrer la réduction de l'effort appliqué par le cylindre lors du laminage d'alliages à haute résistance dans leur état superplastique, le cas du laminage d'un alliage de titane Ti-6Al-4V, dont les paramètres sont donnés au tableau I [11] peut être pris comme exemple.

TABLEAU I

Paramètres de laminage de l'alliage Ti-6Al-4V

\begin{tabular}{|c|c|c|c|c|c|}
\hline Etat de l'alliage & Température ${ }^{\circ} \mathrm{C}$ & Taille de grain $\mu \mathrm{m}$ & $m$ & $\sigma_{0} \mathrm{MPa}$ & $\dot{\varepsilon} \min ^{-1}$ \\
\hline superplastique & $\overline{950}$ & $\overline{6,6}$ & $\overline{0,8}$ & $\overline{110,9}$ & $5 \times 10^{-4}-2 \times 10^{-1}$ \\
\hline conventionnel & 950 & 19,8 & 0,3 & 41,6 & $5 \times 10^{-4}-2 \times 10^{-1}$ \\
\hline
\end{tabular}

Le rapport de l'effort appliqué au cylindre dans le cas conventionnel à celui dans l'état superplastique est donné sur la figure 8 pour différentes vitesses de laminage. Ce rapport décroît lorsque la vitesse de déformation croît. Pour des vitesses de déformation voisines de celles qui sont rencontrées en déformation à chaud, la réduction de l'effort appliqué aux cylindres de laminoir devient négligeable pour l'alliage étudié. En revanche, à des vitesses suffisamment faibles, la réduction de l'effort est plus importante et per-

FIG. 8. - Rapport entre les efforts spécifiques nécessaires pour laminer deux alliages $\mathrm{Ti}-6 \mathrm{Al}-4 \mathrm{~V}$ conventionnels et superplastiques, en fonction de la vitesse des cylindres du laminoir $h_{1}=2 \mathrm{~mm}$, $r=12,5 \%, R=90 \mathrm{~mm}, \mu=0,14$.

[Ratio of roll loads required to roll conventional and superplastic Ti-6Al-4V alloys, versus rolling speed $h_{1}=2 \mathrm{~mm}, r=12.5 \%$, $R=90 \mathrm{~mm}, \mu=0.14$.]

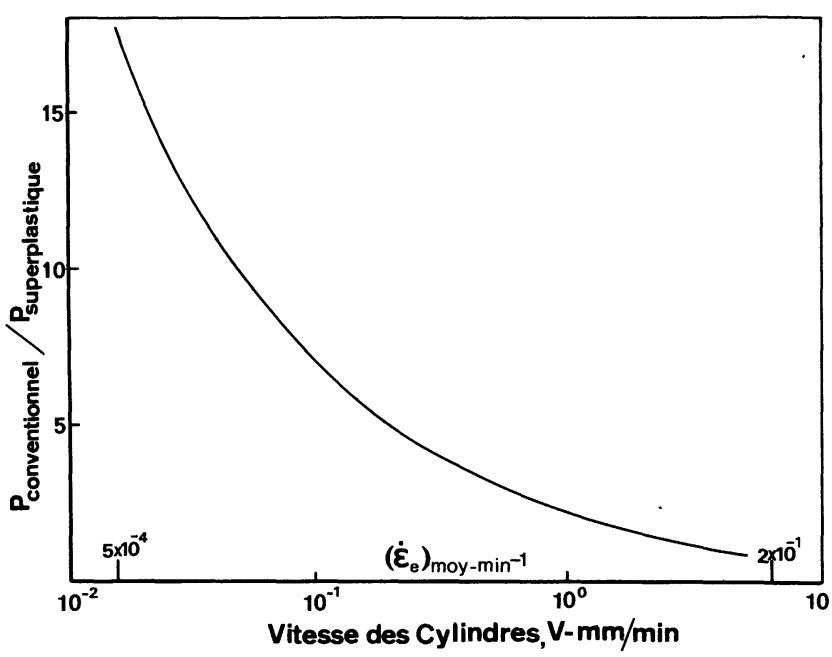


mettrait de laminer des alliages de titane avec de faibles puissances. Cette réduction dans l'effort appliqué aux cylindres permet de laminer des alliages de titane avec un petit laminoir. Cependant, le procédé exige de faire une étude pour optimiser la perte de temps par un gain appréciable de l'effort. On notera aussi que ce procédé exigerait de pouvoir maintenir à température élevée en un temps relativement long les tôles d'alliage de titane au cours du laminage dans des conditions de superplasticité.

Le caractère superplastique temporaire à grande vitesse [7] de certains alliages peut être aussi avantageusement utilisé pour laminer de tels alliages sous des efforts réduits. En effet, certains matériaux peuvent présenter après un fort corroyage ou un important écrouissage suivi d'un recuit, des structures temporaires associées à des grains très fins de taille inférieure à un micron. Aussi, un comportement superplastique peut apparaître d'une manière temporaire dans un matériau conventionnel. Ce comportement superplastique est associé à de grandes vitesses de sollicitation car on sait que pour une contrainte appliquée donnée, la vitesse de déformation dans le domaine superplastique est une fonction fortement décroissante de la taille des grains. Cette superplasticité transitoire permettrait ainsi de laminer des matériaux conventionnels sous des efforts réduits et à des vitesses correspondant aux cadences industrielles.

4. Conclusions. -1 . Une analyse théorique qui prend en compte la sensibilité des matériaux à la vitesse de déformation a permis de calculer l'effort à appliquer aux cylindres lors du laminage de tels matériaux.

2. Les résultats déduits de l'analyse théorique sont en bon accord avec ceux déterminés expérimentalement lors du laminage de l'alliage superplastique $\mathrm{PbSn}$ à différentes vitesses.

3. Le laminage des alliages dans des conditions de superplasticité permet un gain important dans les efforts à appliquer aux cylindres du laminoir. Cependant ceci impose des vitesses de laminage extrêmement lentes, ce qui rend inacceptable le laminage d'alliages conventionnels dans leur état superplastique. Seulement pour les alliages difficiles à laminer dans leur état non superplastique, on peut avoir intérêt à compenser la perte de temps par une forte réduction des efforts à appliquer.

4. Les matériaux qui présentent un comportement superplastique temporaire à grande vitesse, peuvent être laminés à des cadences industrielles tout en réduisant considérablement les efforts à appliquer aux cylindres.

Remerciements. - Les auteurs expriment leur reconnaissance aux organismes (C.N.R.S., D.G.R.S.T.) qui ont soutenu leurs recherches dans le domaine de la superplasticité et à l'Université du Caire pour avoir permis l'utilisation de son Centre de Calcul.

Annexe. - La vitesse de déformation équivalente compte tenu de la constance du volume et du laminage en déformation plane s'exprime :

$$
\dot{\varepsilon}_{\mathrm{e}}=\frac{2}{\sqrt{3}} \dot{\varepsilon}_{h}=\frac{2}{\sqrt{3}} \frac{1}{h} \frac{\mathrm{d} h}{\mathrm{~d} t} .
$$

En considérant la géométrie de l'espace compris entre les cylindres, on voit facilement que la vitesse de déformation locale équivalente [1]

$$
\dot{\varepsilon}_{\mathrm{e}}=\frac{4 V_{\mathrm{N}} h_{\mathrm{N}}}{\sqrt{3 R}} \frac{\sqrt{h-h_{2}}}{h^{2}} .
$$

En considérant les conditions d'équilibre d'un élément de volume situé entre les cylindres, on obtient en faisant intervenir les forces horizontales l'équation bien connue de Von Kàrmàn :

$\frac{\mathrm{d}(p h)}{\mathrm{d} \varphi}=2 S R(\sin \varphi \pm \mu \cos \varphi) \begin{aligned} & + \text { côté sortie } \\ & - \text { côté entrée }\end{aligned}$

Dans cette équation est supposé constant le coefficient $\mu$ de frottement de type Coulomb sur l'arc de contact entier. En établissant l'équation (A.3), il a été admis que la contrainte horizontale $p$ est distribuée uniformément sur les faces verticales de l'élément de volume considéré, comme le suppose la méthode des tranches.

L'angle de contact $\varphi$ étant généralement faible, les approximations $\sin \varphi=\varphi$ et $\cos \varphi=1$ peuvent être faites sans une perte importante de précision. La géométrie de l'arc de contact permet de plus de déduire la variation de $h$ avec $\varphi: h=h_{2}+R \varphi^{2}$. Avec ces approximations, l'équation (A.3) devient :

$$
\frac{\mathrm{d} p}{\mathrm{~d} \varphi}+\frac{2 R \varphi}{\left(h_{2}+R \varphi^{2}\right)} p=\frac{2 R(\varphi \pm \mu)}{\left(h_{2}+R \varphi^{2}\right)} S .
$$

Ainsi que l'a souligné Alexander [12] une erreur très faible est introduite si l'on suppose de plus que la pression normale $S$ et la contrainte horizontale $p$ sont des contraintes principales. La contrainte équivalente est alors donnée par l'expression :

$$
\sigma_{\mathrm{e}}=\frac{\sqrt{3}}{2}(S-p) .
$$

Une relation entre $S$ et $p$ peut être obtenue à partir de la loi de comportement du matériau (2). En substituant à $\dot{\varepsilon}_{\mathrm{e}}$ leurs valeurs respectives, données par les relations (A.2) et (A.5) :

$$
S=p+K \sigma_{0}\left(h-h_{2}\right)^{m / 2} h^{-2 m}
$$


où

$$
K=\frac{2}{\sqrt{3}}\left(\frac{4 V_{\mathrm{N}} h_{\mathrm{N}}}{\dot{\varepsilon}_{0} \sqrt{3 R}}\right)^{m}
$$

Le paramètre $K$ dépend évidemment de la vitesse, de la géométrie du processus de laminage et de la sensibilité à la vitesse du matériau laminé.

L'équation différentielle de base pour la contrainte horizontale $p$ est obtenue à partir des équations (A .4) et (A.6) :

- côté sortie :

$\frac{\mathrm{d} p}{\mathrm{~d} \varphi}-\frac{2 \mu R}{h_{2}+R \varphi^{2}} p=$

$=2 K \sigma_{0} R^{1+m / 2}\left(h_{2}+R \varphi^{2}\right)^{-2 m-1}(\varphi+\mu) \varphi^{m}$

- côté entrée :

$\frac{\mathrm{d} p}{\mathrm{~d} \varphi}+\frac{2 \mu R}{h_{2}+R \varphi^{2}} p=$

$=2 K \sigma_{0} R^{1+m / 2}\left(h_{2}+R \varphi^{2}\right)^{-2 m-1}(\varphi-\mu) \varphi^{m}$.

Les équations différentielles (A.8) ne sont pas susceptibles d'intégration analytique (même dans le cas où le coefficient $m$ est nul). Elles ont donc été intégrées numériquement en prenant pour conditions limites $p$ égal à 0 lorsque $\varphi$ est nul et égal à $\varphi_{1}$. Une fois la distribution des contraintes horizontales $p(\varphi)$ obtenue, la distribution des pressions normales au cylindre $S(\varphi)$ a pu être déterminée grâce à l'équation (A.6).

En négligeant la contribution des forces tangentielles dues au frottement et au remplacement $\cos \varphi$ par 1, l'effort appliqué au cylindre par unité de largeur, effort spécifique sur le cylindre, est donné par l'expression :

$$
\begin{aligned}
P / \frac{2 \sigma_{0}}{\sqrt{3}} & =\left(\frac{4 V_{\mathrm{N}} h_{\mathrm{N}}}{\sqrt{3 R} \dot{\varepsilon}_{0}}\right)^{m} \times \\
& \times R\left[\int_{\varphi_{\mathrm{N}}}^{\varphi_{1}}\left(\frac{S}{K \sigma_{0}}\right) \mathrm{d} \varphi+\int_{0}^{\varphi_{\mathrm{N}}}\left(\frac{S}{K \sigma_{0}}\right) \mathrm{d} \varphi\right]
\end{aligned}
$$

Les équations (A.8) et (A.9) permettent d'estimer la force à appliquer aux cylindres de laminoir dans des conditions données de laminage, à condition de connaître le coefficient $m$ de sensibilité du matériau à la vitesse de déformation.

On peut écrire une équation du couple spécifique exercé sur le cylindre en négligeant la déformation de ce dernier

$$
T=\mu K \sigma_{0} R^{2}\left[\int_{\varphi_{\mathrm{N}}}^{\varphi_{1}}\left(\frac{S}{K \sigma_{0}}\right)^{-} \mathrm{d} \varphi-\int_{0}^{\varphi_{\mathrm{N}}}\left(\frac{S}{K \sigma_{0}}\right)^{+} \mathrm{d} \varphi\right]
$$

\section{Bibliographie}

[1] Ford, H. et Alexander, J. M., J. Inst. Met. 92 (1963-64) 397.

[2] SIMS, R. B., Ibid., 168 (1954) 191.

[3] Denton, B. K. et Crane, F. A., J. Iron Stat. Instrum. (1972) 606.

[4] El-Kalay, A. K. et Sparling, L. G. M., Ibid. (1968) 152.

[5] RAGAB, A. R. et DunCAN, J. L., Int. Symposium on Foundations of Plasticity (Warsaw) édité par A. Sawczuk, 1972, p. 271.
[6] Ragab, A. R. et Baudelet, B., Proceedings of 18th Int. M.T.D.R. (Imperial College, London) 1977, p. 109.

[7] Baudelet, B., Met. Mater. (1974) 117.

[8] Baudelet, B. et Suery, M., J. Mat. Sci. 7 (1972) 512.

[9] UNDER wOOD, L. R., The rolling of Metals (Chapman and Hall Ltd) 1952, Vol. 1.

[10] ARnold, R. R. et Whitton, P. W., Proc. Inst. Mech. Eng. 173 (1959) 241.

[11] Lee, D. et BaCkofen, W. A., Trans. AIME 239 (1967) 1034. [12] Alexander, J. M., Proc. R. Soc. A 326 (1972) 535. 